\title{
SUBSTANTIATION OF DESIGN AND PARAMETERS OF ROTARY HARROW FOR PRE- EMPLOYMENT PROCESSING RIDGE PLANTING OF POTATOES
}

\author{
Marat Salimzyanov $^{1}$, Vladimir Pervushin ${ }^{1}$, Nicolai Kasimov ${ }^{1}$, Marat Kalimullin ${ }^{2}$ \\ ${ }^{1}$ Izhevsk State Agricultural Academy, Russia; ${ }^{2}$ Kazan State Agrarian University, Russia \\ salimmar@mail.ru, pervushin54@mail.ru,nikolakas@list.ru,marat-kmn@yandex.ru
}

\begin{abstract}
The article presents the results of studies in the field of kinematics of the interaction of the elements of a rational working body along the ridge of potato soil to substantiate its parameters. Caring for potato plants is especially important in the initial (pre-emergence) period of the growing season, since at this time the formation of the future crop and the need or failure of subsequent mechanical and chemical treatments occur. Existing passive tools do not provide high-quality destruction of weeds, reactive-active ones do not sufficiently process the tops of the ridge, and milling (active) tools lead to excessive soil dispersion, have a complex drive, high energy and metal consumption. The purpose of the study is to substantiate the geometric scheme and parameters of a rotary harrow for weed destruction during pre-emergence processing of potato comb planting. According to the results of theoretical and field research and parameters of a rotary harrow that fits the profile of the ridge and rolls over the surface of the ridge, which can completely comb and drag weeds along the ridge, destroy the connection of their root neck with the soil, as well as dump them on top of the soil in a certain mode of operation. Namely, the points of the outer rim of the harrow along the row spacing with radius $\mathrm{R}$ will describe an ordinary cycloid, and have a kinematic operating mode indicator $\lambda=1$, and all points lying inside the circle of the harrow rim with a small radius $r_{i}$, a shortened cycloid and have a kinematic operating mode indicator $\lambda<1$ (points of scrapers-slats).
\end{abstract}

Keywords: rotary harrow, pre-emergence processing, parameters, operating mode, kinematic indicator, speed.

\section{Introduction}

The demand for ecological products grown using the technology of minimal chemical treatments is growing from year to year [1]. Crop productivity is the result of the interaction of many factors and conditions, the most important of which are: soil fertility, plant nature, soil and climatic conditions and mechanized tillage technology [2].

Care of potato plants is especially important in the initial (pre-emergence) growing season, since at this time the formation of the future crop occurs, the germination of most early weeds, which will affect the need or failure of subsequent mechanical and chemical treatments, and the cost and price of the product. In addition, the soil crust makes it difficult to germinate, damages the root system of plants, increases the evaporation of moisture, which requires mechanical tillage [3].

Pre-emergence harrowing should be completed when the seedling length reaches $10-15 \mathrm{~mm}$, since after the formation of the primary root system and adhesion of the sprouts to the soil, the harrows can cause them to break off and thin out the crops, which reduces the further efficiency of mechanical processing after germination.

Since the pre-emergence period of potatoes is quite long (15-20 days), and during this period a lot of early weeds enter, so pre-emergence harrowing is carried out - twice with an interval of 5-8 days [4]. Existing passive tools do not provide high-quality destruction of weeds, reactive-active ones do not sufficiently process the tops of the ridge, and milling (active) tools lead to excessive soil dispersion, have a complex drive, high energy and metal consumption.

The purpose of the study is to substantiate the parameters and operating modes of the rotary harrow for pre-emergence processing of the potato ridge planting and create favorable conditions for its growth, due to surface cultivation, weed control and improvement of the water-air balance of the soil surface.

Scientists Vasilenko P. M., Kanarev F. M., Matyashin Yu. I., Abdrakhmanov R. K., Zimmerman M. Z., Yatsuk, E. P. and others were engaged in the creation of rotary tools and machines for potato cultivation.

The explanation of the parameters of rotary harrows that ensure their high productivity is relevant and requires taking into account the influence of factors: humidity, type, density and structure of the soil, norms of agricultural requirements, depth of processing, rotation speed, etc [5]. 


\section{Materials and methods}

In the course of theoretical studies and measuring the profile of the ridges during the ridge planting of potatoes, a rotary harrow was designed to fit the profile of the ridge and passively rolling around the circumference of the rim of the soil cultivators, the points of which describe the trajectory of an ordinary cycloid.

Theoretically, the ridge profile can be taken in the form of a trapezoid, which is characterized by a height $H$, a width $b$ at the upper base and a width $B$ of the lower base equal to the row spacing (Fig. 1).

According to the All-Russian Research Institute of Potato Farming (NIIKH), the parameters of the ridge before leaving had the following values shown in Table 1.

\section{Parameters of the ridge during the care of planting potatoes}

Table 1

\begin{tabular}{|c|c|}
\hline Indicators & Values, $\mathbf{c m}$ \\
\hline Height, $\mathrm{H}$ & $12-16$ \\
\hline Width at the base, $\mathrm{B}$ & 70 \\
\hline Width on top, $\mathrm{b}$ & $5-10$ \\
\hline
\end{tabular}

The trajectory of any circumference of a rotary harrow is a cycloid, which is the result of their rotation around the axis of the harrow with a speed $v_{p}$ and translational motion with a tractor with a speed $v_{m}\left(v_{t}\right)$ and in a fixed coordinate system, is described in the parametric form of the equations: [4; $6 ; 7]$ :

$$
\begin{gathered}
x=v_{M} \cdot t+r_{i} \cdot \sin (\omega \cdot t), \\
y=r_{i} \cdot \cos (\omega \cdot t),
\end{gathered}
$$

where $v_{m}$-machine(tractor) translational speed $\left(v_{t}\right), \mathrm{m} \cdot \mathrm{s}^{-1}$;

$t$ - time, s;

$r_{i}-$ radii of the circles $(r \ldots R), \mathrm{m}$;

$\omega$ - angular speed of the harrow), radian $\cdot \mathrm{s}^{-1}$.

The period of time $\mathrm{T}$ for a complete revolution of the circle is:

$$
T=2 \pi / \omega=1 / v,
$$

where $v$-rotation frequency, $\mathrm{s}^{-1}$.

It is known that the center of the circle of the harrow (p.O) moves with a constant translational speed of the tractor (machine) $v_{m}$ and over a period of time of a full $\mathrm{T}$ revolution passes a path equal to:

$$
\begin{aligned}
& S=2 \pi r_{i}, \\
& S=v_{m} \cdot T,
\end{aligned}
$$

Linear (circumferential) speed of circles of different radii $(r \ldots R)$ :

$$
v_{p i}=\omega \cdot r_{i},
$$

The following assumptions are used.

1. The rotary harrow rolls along the ridges, that is, it moves progressively and simultaneously rotates around its central axis (point $O$ ), relying on planks and rims on the soil without slipping (slipping).

2. The speed of the tractor (machine), and therefore the drum, is taken within $6-9 \mathrm{~km} \cdot \mathrm{h}^{-1}(1.7-$ $2.5 \mathrm{~m} \cdot \mathrm{s}^{-1}$ ) - according to agricultural requirements.

\section{Results and discussion}

The rotary harrow is hollow and through geometric figure composed of an internal horizontal cylinder 1 of small radius and outside of two truncated horizontal cones 2 , the direction of which is outward from a small radius to a large radius (Fig. 1). Considering structurally, the outer 
circumference of the cylinder and the cones are vertical rims 3 interconnected by horizontal scrapersstrips 4 by welding.

The geometric design parameters of the rotary harrow are determined by the size of the ridge profile, the row spacing of the plants, the depth of the tubers and the location of the rotary harrow relative to each other in the direction of travel, namely in a checkerboard pattern, which will ensure overlapping trajectories of the extreme rims of the cones of the rotational harrow along the row and repeated destruction and loosening soil in it [1].

Progressive potato cultivation technology is cultivated on $75 \mathrm{~cm}$ aisles. In this case, we can write that the width of the harrow $\mathrm{B} 1$ is equal to the width of the row spacing: $B_{1}=B$.

a)

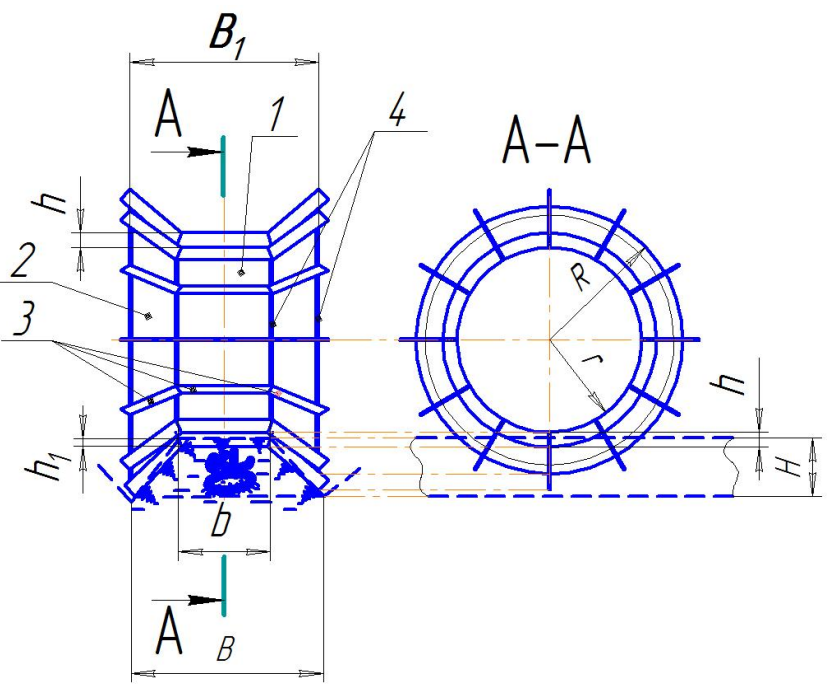

b)

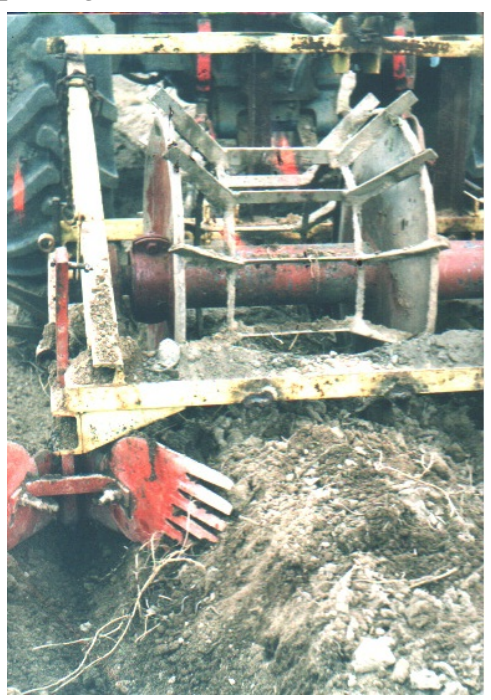

Fig. 1. Geometric parameters (a) and photo (b) of the rotary harrow and comb (ridge):

1 - cylinders; 2 - cones; 3 - scrapers-strips; 4 - rims

Our field studies after planting potatoes showed an average ridge height of $10-12 \mathrm{~cm}$ with a row spacing of $70 \mathrm{~cm}$, and between rows of $75 \mathrm{~cm}$ with different technology $-23-25 \mathrm{~cm}$. We accept the average value of $17.5 \mathrm{~cm}$.

The obtained geometric parameters of the rotary harrow are summarized in Table 2.

Table 2

\section{Geometric parameters of rotary harrow}

\begin{tabular}{|l|c|}
\hline \multicolumn{1}{|c|}{ Indicators } & Values, $\mathbf{~ m m}$ \\
\hline Harrow width for row spacing $70 \mathrm{~cm} ; 75 \mathrm{~cm}, B_{1}$ & $700 ; 750$ \\
\hline Inner cylinder width, $b$ & $100-120$ \\
\hline Height of the scraper bar (fingers), $h$ & $50-60$ \\
\hline Depth of the working element in the soil, $h_{1}$ & $20-30$ \\
\hline Ridge height for row spacing $70 \mathrm{~cm} ; 75 \mathrm{~cm}, H$ & $155 ; 175$ \\
\hline Outdoor ratio $R$ and inner $r$ radii & $R=r+H$ \\
\hline
\end{tabular}

Having more clearly examined the trajectory of motion in the form of cycloid scrapers-strips of harrows around a circle of radius $(r \ldots R)$ under the condition of complete destruction of weeds and destruction of the soil crust of the crest surface, depending on the kinematic mode of operation $\lambda$, we will be able to explain the radius and translational speed.

$$
\lambda_{i}=v_{p i} / v_{m}=\left(\omega \cdot r_{i}\right) / v_{m},
$$

From an economic point of view, the radius of the drum should be as small as possible in order to reduce dimensions and weight and reduce the cost of construction. On the other hand, the speed of the unit should be as high as possible in order to achieve high productivity of the process of caring for potato plants.

For clarity, let us consider in the transverse plan the full rotation of the boron, dividing the segment of movement into equal intervals of 12 parts and from the center of $O_{i}$ with radii $r, r 1, R$ at an 
angle of $30^{\circ}\left(360^{\circ} / 12\right)$, we get the extreme points of the corresponding radii, connecting them we get different types of trajectory of cycloids.

Theoretically, three options for rolling the harrow are possible [4].

1. Along the circumference of the inner cylindrical part of the harrow along the top of the ridge $(r)$;

2. Around the circumference of the conical part along the guide rails $(r \ldots R)$;

3. Around the circumference of the outer rim of the soil row spacers $(R)$.

In the first case, all points lying on the cylindrical part $(r)$ of the harrow describe the normal cycloid and the indicator of the kinematic mode of operation $\lambda=1$. All other points of the harrow that guide the cone and outer rim lying at large radii $(r \ldots R)$ describe elongated cycloids and their kinematic mode of operation $\lambda>1$ (Fig. 2).

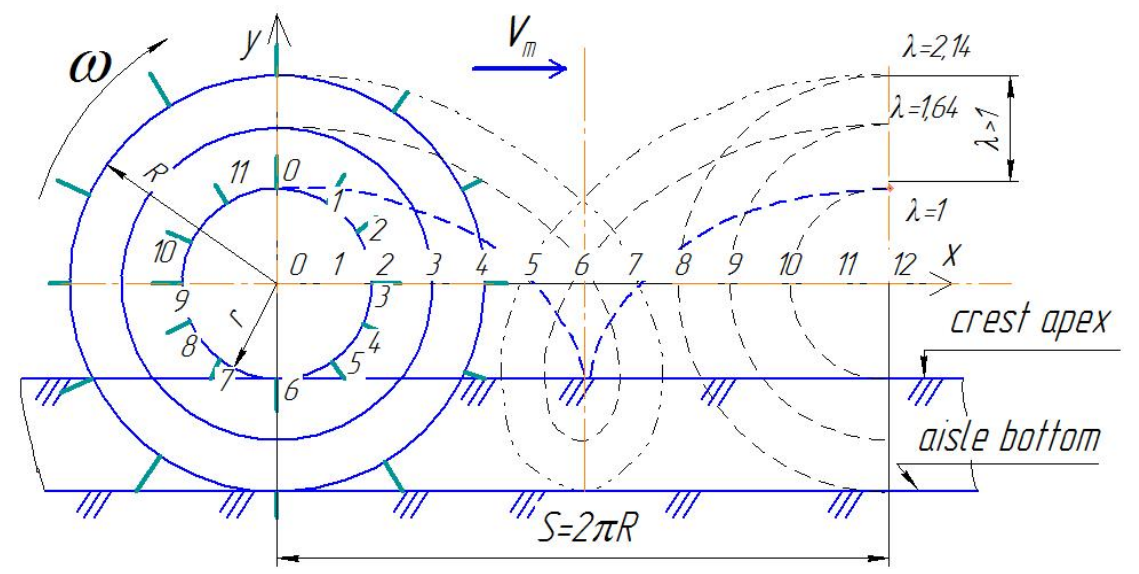

Fig. 2. Rolling the harrow on the circumference of the rotary part of the harrow

In the second case, when the harrow rolls along the conical part $(r \ldots R)$, all points of the harrow lying on a smaller radius $(r)$ will describe a shortened cycloid (elongated) and their kinematic mode indicator $\lambda<1$, and points lying on a larger radius $(R)$ will describe an elongated cycloid and have kinematic mode indicators $\lambda>1$ (Fig. 3).

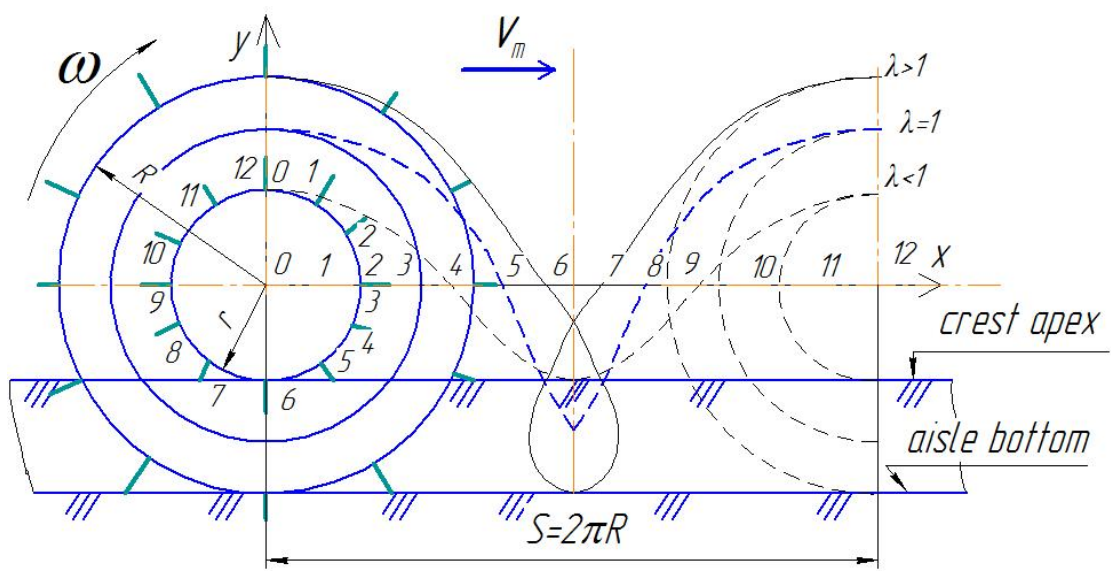

Fig. 3. Rolling the harrow on the circumference of the conical part of the harrow

In the third case, when the harrow rolls along the outer rim of the soil hooks at a greater radius $(R)$ along the row spacing, all its points describe an ordinary cycloid and its kinematic operating mode indicator $\lambda=1$. And other points of the harrow lying on the radius $r \ldots R$, the guides of the cone and cylinder along the top of the ridge describe a shortened cycloid (elongated) and their kinematic operating mode indicator $\lambda<1$ (Fig. 4).

Taking the circumferential speed $\mathrm{vp}$ over the external radius $\mathrm{R}$ of the harrow equal to the translational speed of the tractor (machine)vm and taking into account expressions (4),(5),(7) multiplying by the circumferential velocity vp when converting we get the following [4]:

$$
S=2 \pi R / \lambda \text {. }
$$




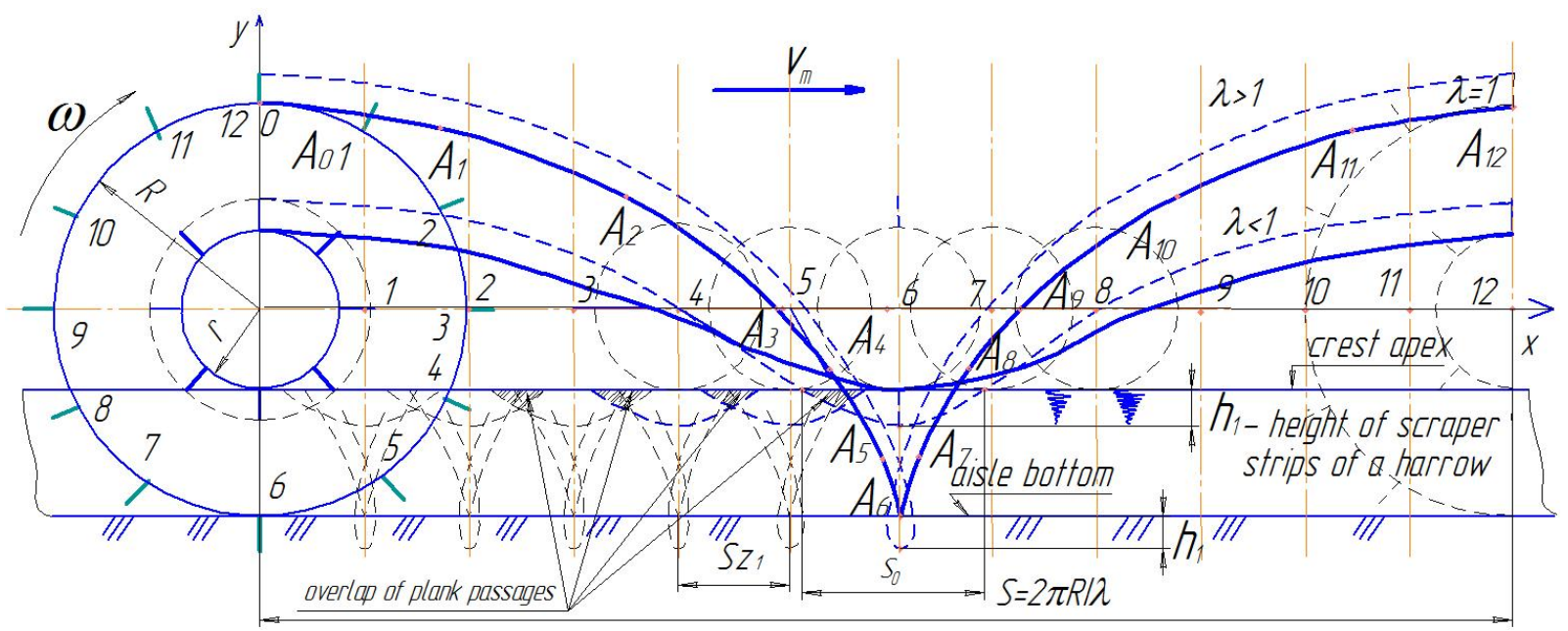

Fig. 4. Absolute trajectory of movement of the initial points of the rotary harrow under the condition of rolling along the outer rim along the row spacing

Substituting expression (3) in (5) and equating to expression (8), we express the radius of the outer rim, we get a dependence on the translational speed, rotation frequency and kinematic index:

$$
R=v m /(2 \pi \cdot v \cdot \lambda),
$$

The rotation frequency is determined from the following relations: the ratio of the trajectory outlined by an ordinary (normal) cycloid equal to $L=8 R$ to the time of its passage of points $t$ is equal to the ratio of the working stroke segment $S=2 \pi R$ passed by the boron for a full revolution to the time period $T$, that is:

$$
8 R / t=2 \pi R / T,
$$

Substituting the formula (3) in this expression, we will express the rotation frequency

$$
v=4 /(\pi \cdot t),
$$

So, for one second the rotary harrow at $\lambda=1$ approximately makes 1.274 turns.

Based on this, the outer radius of the rotary harrow is determined by the expression providing the maximum translational speed for agricultural demand (9):

$$
R=2.5 /(2 \cdot 3.14 \cdot 1.27 \cdot 1)=0.313 \mathrm{~mm}
$$

So, the radius of the harrows rolling over the top of the ridge will be: $r=R-H=0.158 \mathrm{~mm}$.

This option is most favorable and effective, in which the points located at a smaller radius of the harrow $(r . . . R)$ guide the cone and the inner part of the cylinder, make shortened (elongated) cycloids $\lambda<1$ and at the same time drag (loosen) the upper surface layer of the soil forward, thereby raising weeds in the phase of the white thread to the soil surface (Fig. 4). Let us look at this option further to explain this and the number of necessary scrapers-slats.

Planks located at a radius $r \ldots R$ across the row above the top and the side of the ridge at the moment of contact with the soil begin to enter the soil at an acute angle (the beginning of segment $S 0$ ), which corresponds to the position of the center harrow at point $O 4$, then in the middle of the working stroke take a vertical position (point $O 6$ ), and at the end of the working stroke $O 8$ break away from the soil surface (end of segment $S 0$ ) at an obtuse angle. Thus, in the first half of the working stroke (SO/2), the scraper picks up soil and weeds, and in the second - pulls them and throws them off, which is quite favorable for the technological process of pulling weeds and loosening the soil. As can be seen from the graph in Fig. 4, 12 planks are enough to partially overlap the planks' working moves of each other (shaded), as confirmed by field experiments.

Considering the moisture of the soil due to sliding of the harrows and the need to block the passages (SO) of the scrapers (see Fig. 4), the number of scrapers of the slats is 1,5 times larger:

$$
m \approx 1.5 S / S_{0} \text {, }
$$


During field studies with pre-processing of potatoes with a row spacing of $70 \mathrm{~cm}$, the rotary harrow for weeds with the following settings and modes was used: width of the harrow $B_{1}=700 \mathrm{~mm}$, with a radius at the top of the ridge $r=155 \mathrm{~mm}$ and a radius at the aisle $R=315 \mathrm{~mm}$, length and height of scrapers $l=100 \mathrm{~mm}, h=50 \mathrm{~mm}$, in the amount of 12 pcs., with a forward speed of $v_{m}=2.5 \mathrm{~m} \cdot \mathrm{s}^{-1}$, the resulting small-sized soil structure of up to $10 \mathrm{~mm}$ is more than $60 \%$ and lumps of more than $50 \mathrm{~mm}$ are not detected with high weediness of more than 100 pieces per $\mathrm{m}^{2}$, the number of sprouted weeds after processing is 3.4, which corresponds to $97 \%$ of their death.

\section{Conclusions}

In the course of theoretical and field research, the following conclusions were obtained:

1. For pre-emergence processing of potato ridge plantings, a rotary harrow has been developed that fits the profile of the ridge and passively rolls along the outer circumference of the rim of a large radius $R$ on the row spacing of 70 and $75 \mathrm{~cm}$.

2. A lightweight rotary harrow is a hollow and through geometric figure consisting of an internal horizontal cylinder of small radius $r$ and two truncated horizontal cones outside, directed outward from a small radius $r$ to a large radius R. Structurally, the cylinder and cones are outer circles of vertical rims, connected by horizontal scrapers-strips by welding.

3. Parameters and modes of operation of rotary harrows: width of the harrows $B_{1}=700 \mathrm{~mm}$, with an internal radius at the top of the ridge $r=155 \mathrm{~mm}$ and the radius of the row spacing $R=315 \mathrm{~mm}$, length and height of the strip scrapers $l=100 \mathrm{~mm}$ and $h=50 \mathrm{~mm}$, in the amount of 12 grand., forward speed $v_{m}=2.5 \mathrm{~m} \cdot \mathrm{s}^{-1}$.

4. Kinematic indicator operation mode, the appropriate $\lambda=1$ rotary harrow rolls on the outer rim of the aisle radius $R$, which corresponds to the normal to the cycloid motion of the straps, other straps which are located on the guide cone and the cylinder and over the sidewall and the top ridge on a smaller radius $(r<R)$ do a shortened cycloid $\lambda<1$, and at the same time pull the straps to drag (loosen) the top surface layer of soil forward, thereby raising the weeds in the phase of white threads on the soil surface.

\section{References}

[1] Osowski J. Innovation and optimization in potato cultivation protection. Zywnosc. Nauka. Technologia. Jakosc/Food. Science Technology. Quality. 25. pp. 42-55. DOI: 10.15193/zntj/2018/117/258.

[2] Yaropud V., Volyk B. Rationale for the needle disc design of a rotary harrow with the analysis of the structure of the body of a biological analogue. Vibrations in engineering and technology. 2019, pp. 56-64. DOI: 10.37128/2306-8744-2019-4-7.

[3] Sheichenko V., Dudnikov I., Shevchuk V., Kuzmych A. Research of surface-plane and spacedeep interaction of needle with soil. Agricultural machinery. Proceedings. "Uses of machines. Innovative technologies. Conserving soils and water." VII International Scientific congress. Vol.2. Scientific-Technical Union of Mechanical Engineering industry 4.0. 26-29 JUNE 2019, BURGAS, BULGARIA, pp. 93-96.

[4] Первушин В.Ф., Салимзянов М.З., Николаев В.А. Кинематические параметры ротационного рыхлителя (Kinematic parameters of a rotary cultivator) Mechanization and electrification agricultural. Journal, 2009, No 6. pp. 37-38.

[5] Hirasawa K., Kataoka T., Kubo T. Prediction and evaluation for leveling performance in rotary tiller. Proc. 4th IFAC Conference on Modelling and Control in Agriculture, Horticulture and Post Harvest Industry, Espoo, Finland, 46, 2013, pp. 315-20.

[6] Kalimullin M.N., Abdrakhmanov R.K., Arkhipov S.M. Rotary haulm chopper parameters development and substantiation for root and tuber crops. International Journal of Applied Engineering Research, Vol. 10, Num. 10 (2015), pp. 25691-25697.

[7] Raparelli T., Pepe G., Ivanov A., Eula G. Kinematic analysis of rotary harrows. Journal of Agricultural Engineering, vol. 976, Num. 1 (2020), 2019, pp.9-14. 